
available under aCC-BY 4.0 International license.

\title{
TITLE: Shank3 influences mammalian sleep development
}

Authors: Hannah Schoch ${ }^{1}$, Elizabeth Medina ${ }^{1}$, Taylor Wintler ${ }^{1}$, Kaitlyn Ford ${ }^{1}$, Kristan G. Singletary ${ }^{1}$ and Lucia Peixoto ${ }^{1 *}$.

\section{Affiliations:}

1. Department of Biomedical Science, Elson S. Floyd College of Medicine, Washington State University, Spokane, Washington, United States

* To whom correspondence should be addressed: Pharmaceutical and Biomedical Science Building \#220, 412 E. Spokane Falls Blvd. Spokane WA 99202. Tel: 509-368-6764, Fax: 509-368-7882. Email:

lucia.peixoto@wsu.edu.

\section{ABSTRACT}

Sleep problems are prevalent in Autism Spectrum Disorder (ASD), can be observed before diagnosis and are associated with increased restricted and repetitive behaviors. Therefore, sleep abnormalities may be a core feature, but the developmental trajectory remains unknown. Previously we showed that adult mice with a truncation in Shank3 (Shank3 ${ }^{\Delta C}$ ) recapitulate the ASD sleep phenotype (Ingiosi et al, 2019). In this study we used longitudinal electro-encephalographic recordings to define, for the first time, changes in sleep from weaning to young adulthood in an ASD mouse model. We show that Shank ${ }^{\Delta C}$ mice sleep less throughout their lifespan and have abnormal responses to increased sleep pressure during a specific developmental period. More specifically, mutants appear unable to rapidly enter sleep, despite preceding periods of prolonged waking. Overall, our study indicates that reduced sleep is a core feature of ASD and suggests that problems falling asleep reflect abnormal development in sleep and arousal mechanisms.

\section{IMPACT STATEMENT}

In this first longitudinal sleep study in an Autism mouse model, we demonstrate that sleeping less is a core feature while problems falling asleep emerge during development.

\section{INTRODUCTION}

Good sleep is a cornerstone for maintaining optimal health. Accordingly, sleep problems, with insomnia as the most common sleep disorder, impact significantly on our physical capabilities and mental health. In individuals diagnosed with Autism Spectrum Disorder (ASD), two thirds have chronic insomnia (defined as persistent problems falling and staying asleep), and $86 \%$ of people with ASD are affected by sleep problems ${ }^{1,2}$. Relative to individuals that don't have ASD, individuals with ASD experience significant delays falling asleep, multiple night awakenings and overall less sleep time ${ }^{3}$. Poor sleep is predictive of the severity of ASD core behavioral diagnostic symptoms such as social skill deficits and stereotyped behavior, worsen with age, and heavily affects the quality of life of individuals and their caregivers. In addition, sleep problems in young children that go on the be diagnosed with ASD are associated with increased 'higher-order' restricted and repetitive behaviors later in childhood ${ }^{4}$ and altered patterns of brain development ${ }^{5}$. Although commonly referred to as a condition co-morbid with ASD, when children are diagnosed with ASD, they often already have a history of sleep problems, sometimes starting as early as the first year of life. Given the well-documented role of sleep in brain development, early-life sleep disruption is likely to contribute to later-life core features of ASD and might even be an indication for early intervention. However, given that the developmental trajectory of sleep problems in ASD precedes diagnosis, their role in ASD etiology remains largely unexplored.

ASD is known to have a strong genetic component including both de novo and inherited gene variations. Nonetheless the same variant can cause different symptoms along a spectrum ${ }^{6}$. This heterogeneity presents challenges for genetic ASD animal models that primarily seek to mimic behavioral phenotypes such as social communication deficits and stereotyped behaviors. In addition, this approach generally fails to capture earlier 
neurodevelopmental processes preceding disease onset, leaving etiology elusive. Sleep, unlike other behavioral phenotypes, can be objectively quantified in mammals and is extremely conserved across the animal kingdom. Therefore, animal models are ideally suited to investigate the relationship between sleep and ASD. However, few studies to date have focused on sleep abnormalities. In earlier work, we reported that individuals with Phelan-McDermid syndrome (PMS), a rare genetic syndrome with high rates of ASD diagnosis, have a sleep phenotype akin to those with ASD. PMS is caused either by loss of the tip of chromosome 22 that includes SHANK3 or a mutation in the SHANK3 gene, which encodes a neuronal junction protein critical for synaptic function. Similar to what we observed in patients, we found that adult mice lacking exon 21 of Shank3 $\left(\right.$ Shank $\left.3^{\Delta C}\right)$ slept less and took longer to fall asleep ${ }^{7}$. Shank ${ }^{\Delta C}$ mice also displayed lower levels of electroencephalographic (EEG) slow wave (i.e. 'delta') activity; a marker of sleepiness, suggesting their sleep was also not of the same quality.

In this study, we set out to characterize sleep ontogenesis in Shank $3^{\Delta \mathrm{C}}$ mice with a view to capture early developmental sleep patterns. To this end, we executed longitudinal sleep recordings starting immediately after weaning into young adulthood. We discovered that mutant mice overall sleep less than WT controls, analogous with observations in infants and toddlers with older siblings with for ASD that go on to be diagnosed. Significantly, we find that Shank $3^{\Delta C}$ mice, fail to reduce sleep latency in response to sleep loss as they get older, a physiological response that develops in WT mice between 24 and 30 days of life. These data identify a developmental time window at which early intervention capable of normalizing aberrant sleep patterns could provide therapeutic benefit. Last, we find features of the EEG spectra that may have potential as an early biomarker of sleep problems in ASD. Overall, our study emphasizes the importance of examining developmental trajectory to understand sleep problems associated with ASD.

\section{RESULTS}

Developing Shank3 ${ }^{\Delta C}$ mice sleep less and show altered diurnal/nocturnal distribution of sleep/wake. To understand how the Shank 3 mutation impacts on sleep architecture, it's important to first understand normal sleep development. Longitudinal EEG recordings in young animals are technically challenging, due to the difficulty placing electrodes and obtaining good signals, therefore few studies have been performed to date. Previous studies established that wild-type (WT) mice by postnatal day 21 exhibit a diurnal/nocturnal (circadian) activity pattern and three distinct states based on EEG: wake, non-rapid-eye movement (NREM) and rapid-eye movement (REM) ${ }^{8,9}$, but how sleep changes after the third postnatal week once the animals are separated from their moms and siblings and into adulthood is not known. The developmental trajectory of sleep in genetic animal models of ASD remains completely unexplored. To define basal characteristics of postnatal sleep, starting at the third postnatal week we performed 24-hour EEG and electro-myographic (EMG) recordings from WT and Shank $3^{\Delta C}$ mice under undisturbed (baseline) conditions (at P23, P29, P44, and P59).

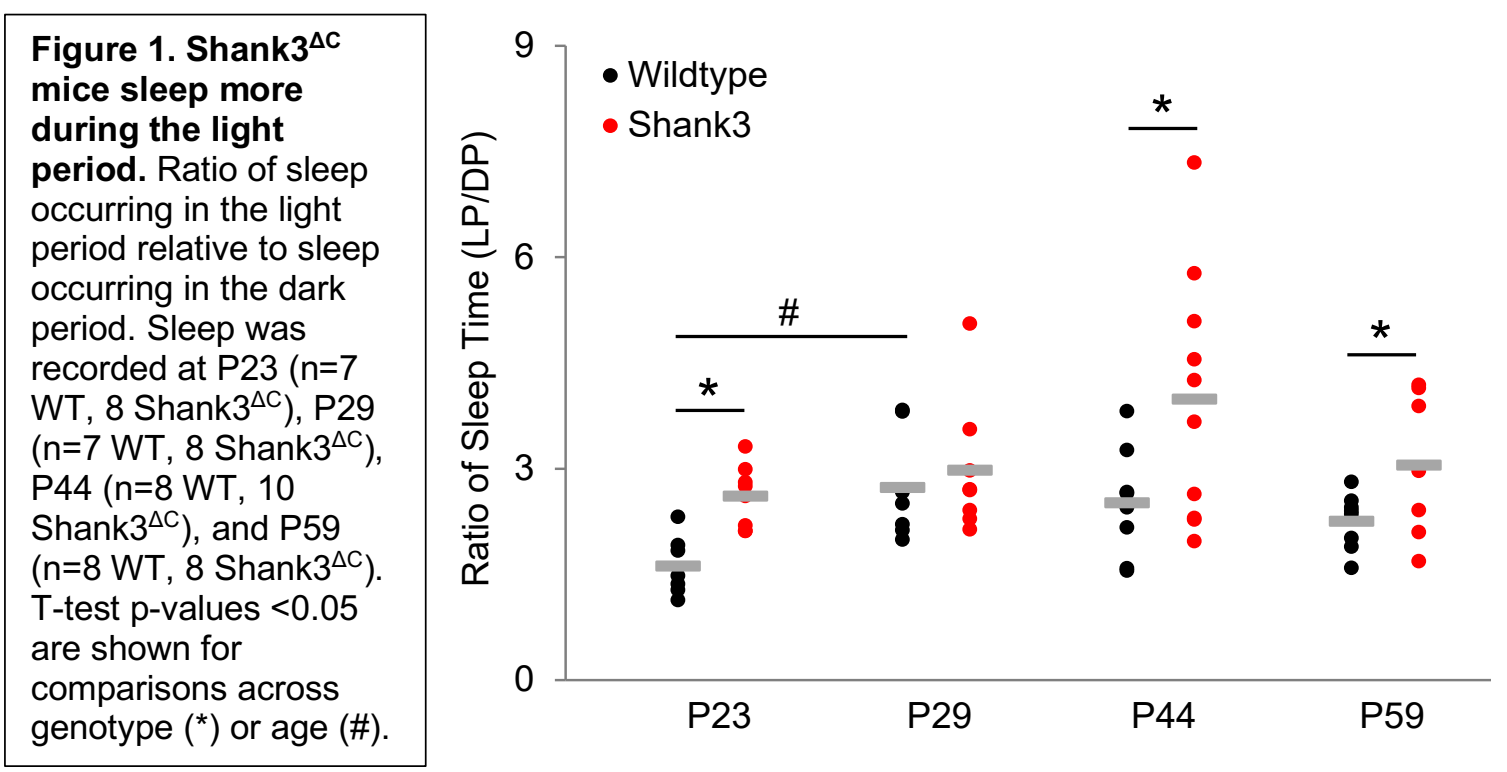


Total Sleep Time (\% of recording time)

\begin{tabular}{lcccc} 
Age & Phase & Wild-type & Shank3 $\Delta C$ & p-value \\
\hline P23 & Light & $55.1 \pm 1.9$ & $57.7 \pm 1.1$ & 0.13 \\
$(n=7,8)$ & Dark & $35.4 \pm 2.2$ & $22.5 \pm 1.2$ & $<0.01$ \\
& $24 h$ & $45.2 \pm 0.5$ & $40.1 \pm 0.7$ & $<\mathbf{0 . 0 1}$ \\
\hline P29 & Light & $63.6 \pm 1.5$ & $58.4 \pm 2.3$ & $\mathbf{0 . 0 3}$ \\
$(n=7,8)$ & Dark & $24.7 \pm 2.2$ & $20.6 \pm 1.7$ & 0.08 \\
& 24h & $44.1 \pm 1.3$ & $39.5 \pm 1.0$ & $<0.01$ \\
\hline P44 & Light & $60.9 \pm 1.8$ & $62.8 \pm 1.2$ & 0.21 \\
(n=8,10) & Dark & $26.1 \pm 2.6$ & $18.5 \pm 2.4$ & $\mathbf{0 . 0 2}$ \\
& 24h & $43.5 \pm 1.4$ & $40.6 \pm 1.0$ & 0.06 \\
\hline P59 & Light & $63.5 \pm 1.1$ & $62.9 \pm 1.6$ & 0.37 \\
(n=8,8) & Dark & $28.9 \pm 1.7$ & $22.5 \pm 2.5$ & $\mathbf{0 . 0 3}$ \\
& 24h & $46.2 \pm 0.9$ & $42.7 \pm 1.3$ & $\mathbf{0 . 0 2}$ \\
\hline
\end{tabular}

Table 1: Shank3 ${ }^{\Delta C}$ mice sleep less throughout their lifespan. Total baseline sleep time during 12 hours of light, 12 $\mathrm{h}$ ours of dark, or across the full day expressed as percent of total recording time (24 hours). Sleep was recorded at postnatal days $23,29,44$, and 59. Postnatal day 23 (P23) $(n=7$ WT, 8 Shank $\left.3^{\Delta C}\right), P 29(n=7$ WT, 8 Shank $\left.3^{\Delta C}\right), P 44(n=8$ WT, 10 Shank $\left.3^{\Delta C}\right)$, P59 $(n=8$ WT, 8 Shank $3^{\Delta C}$ ) mice. $p-$ values shown for genotype comparisons at each age (ttests).

In WT animals, we found that the distribution of total sleep time differed significantly between P23 and P29 mice (combined time in NREM and REM) across the light (LP, hours $0-11$ ) and dark (DP, hours 12-24) periods, measured as the ratio of time asleep in the light period versus the dark period (Figure 1). These data suggest that circadian regulation of sleep/wake continues to develop between P23 and P29. We also found that normal sleep development in adolescence (P44) is characterized by a greater variability in distribution of sleep time across the light-dark periods. Although sleep consolidation in WT mice in the light period is still evolving between P23 and P29, this shift (no change in sleep distribution) is absent in Shank3 ${ }^{\Delta C}$ mice (Figure 1). Thus, based on these data, Shank $3^{\Delta C}$ mice show a precocious development of nocturnal/diurnal sleep organization. During adolescence, the Shank $3^{\Delta C}$ mutation also confers an increased variability in nocturnal/diurnal sleep organization. Despite the fact that Shank $3^{\Delta C}$ mutants spend more time sleeping during the normal inactive phase (light period), they sleep less overall than WT mice at all time points (dark period, and in total) (Table 1).

\section{Shank $3^{\Delta C}$ mice have higher amounts of REM sleep early in life.}

To determine which states are affected by the overall reduction in sleep, we examined time awake, in NREM, and in REM sleep over 24 hours starting at P23. At this developmental time point, mice have acquired $70 \%$ of their maximal brain volume and are equivalent to a 9-month-old infant based on brain size ${ }^{10}$. The role of sleep during this developmental time period is of particular interest given that results from behavioral studies suggest a period of typical development followed by the early post-natal onset of ASD in the latter part of the first year or early second year of life in human infants ${ }^{11}$.

Similar to what we previously reported at P90, increased wakefulness (reduced sleep) in Shank $3^{\Delta C}$ mice is most pronounced during the dark period at P23 ( $p=0.04$; Figure 2A, Figure 2-figure supplement 1). Time spent in NREM is significantly reduced in Shank ${ }^{\Delta C}$ mice in both light and dark periods across development (P23: LP $p>0.01$, DP $p<0.01$; P29: LP $p>0.01$, DP $p=0.04$; P44: LP $p=0.59$, DP $p=0.05$; P59: LP $p=0.04$, DP $p=0.03$; Figure 2B, Figure 2-figure supplement 1 ). Bouts of NREM sleep are also shorter across development in Shank3 ${ }^{\Delta C}$ mice, particularly in the dark period (Figure 2-figure supplement 2). Through postnatal development, and relative to WT mice, Shank $3^{\Delta C}$ mice show an increase in REM from P23 to P59 (P23 p<0.01; P29 p=0.04; P59 p=0.04; Figure 2C, Figure 2 - figure supplement 1 ) during the light period. Increased REM sleep at P23 and P29 in Shank3 ${ }^{\Delta C}$ mice is driven by an increase in entries into REM during the light period (P23 p>0.01, P29 p=0.03; Figure 2 - figure supplement 2). Together, these findings suggest that state-specific sleep differences in Shank $3^{\Delta C}$ mice are dynamic and emerge early post-weaning. 


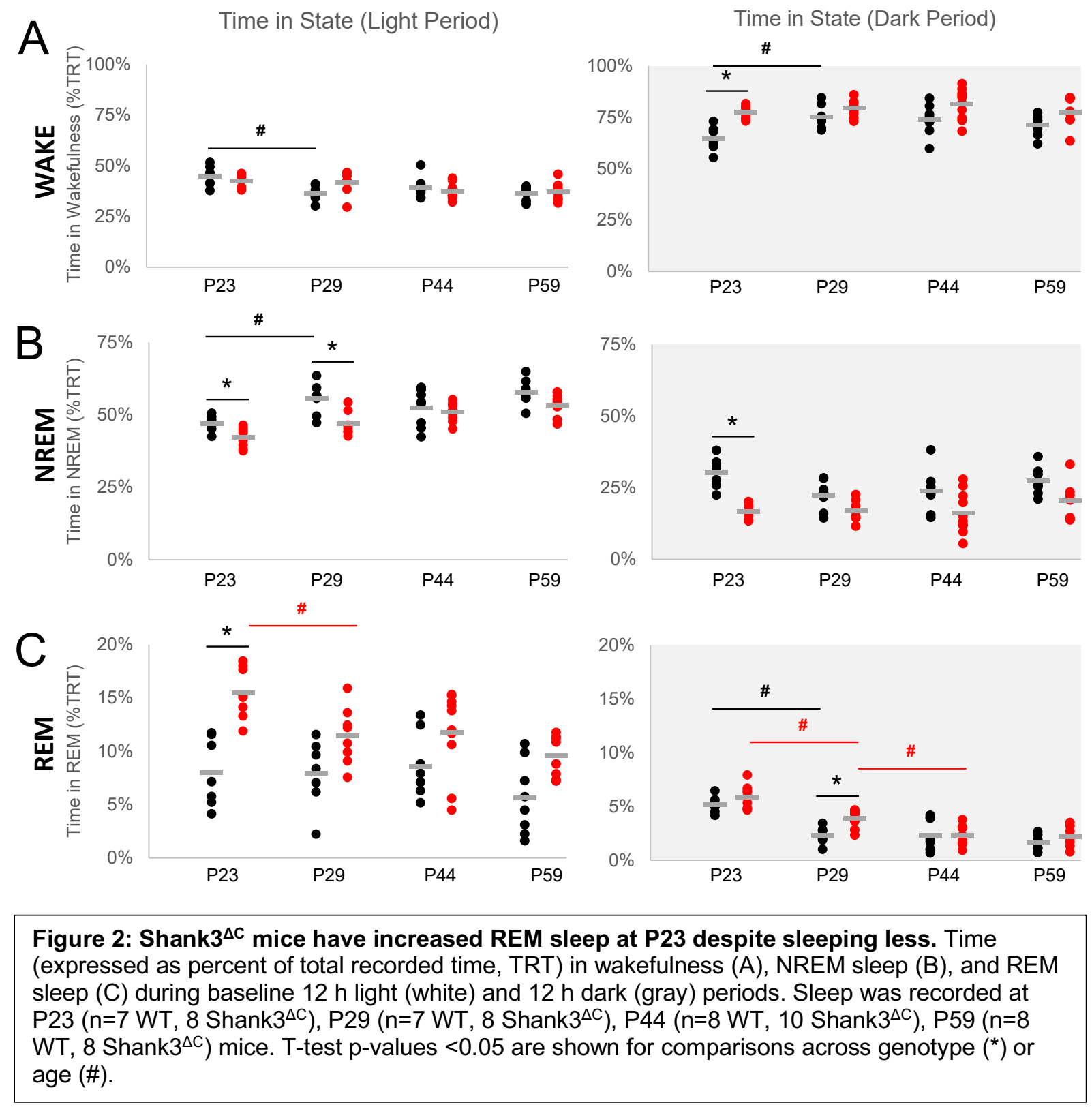

\section{Spectral power in all brain states changes across development differentially in Shank $3^{\Delta C}$ and WT mice.}

Spectral properties of the rodent cortical EEG waveform are developmentally regulated in a state-specific manner $8,9,12$, and our data in WT animals supports this observation (Figure 3). However, although the power in the theta frequency range $(5-10 \mathrm{~Hz})$ is gradually reduced through P23 to P59 stages in WT in both the wake and REM spectra, this trend is not present in Shank $3^{\Delta C}$ mice, in which the spectral power at this frequency remains the same for all time points (Figure $3 A$ and $3 C$ ). In adult Shank $3^{\Delta C}$ mice, we reported that EEG slowwave delta $(0.5-4 \mathrm{~Hz})$ activity in NREM sleep is reduced ${ }^{7}$. Here, we demonstrate that this feature results from a progressive loss of NREM delta activity throughout postnatal development (Figure 3B, Figure 3- figure supplement 1). In WT mice, NREM delta activity is relatively stable across the same period, showing only a small reduction between P23 and P29. We also found spectral differences during REM sleep in Shank $3^{\Delta C}$ mice, specifically a rightward shift in the peak of theta $(5-10 \mathrm{~Hz})$ frequency activity and a reduction in overall delta activity (Figure $3 \mathrm{C}$ - figure supplement 1 ). 

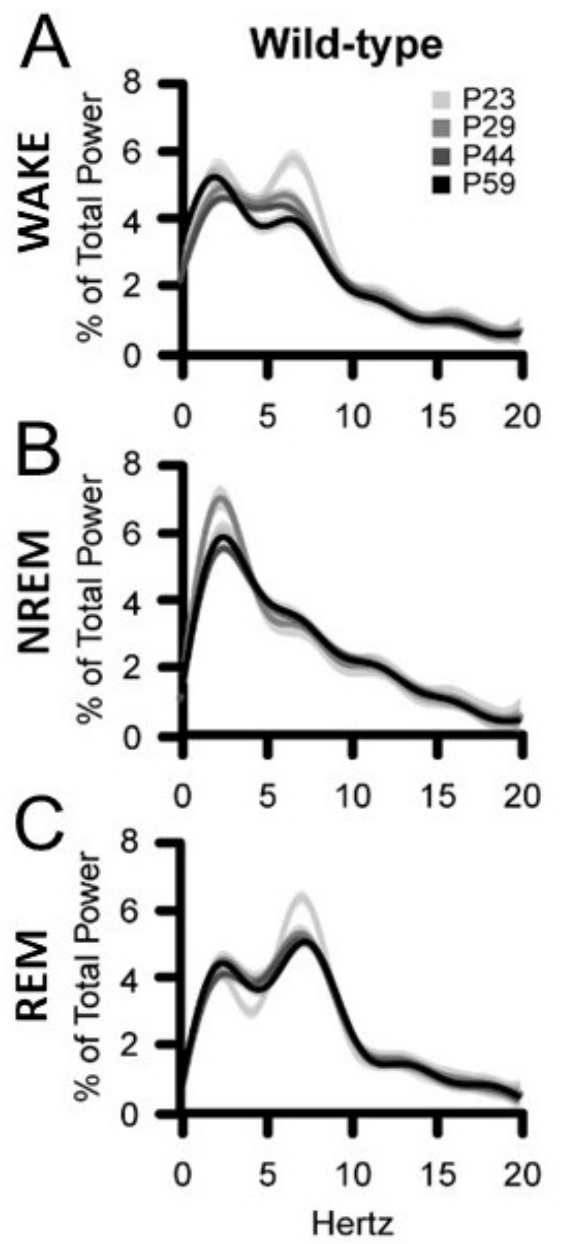
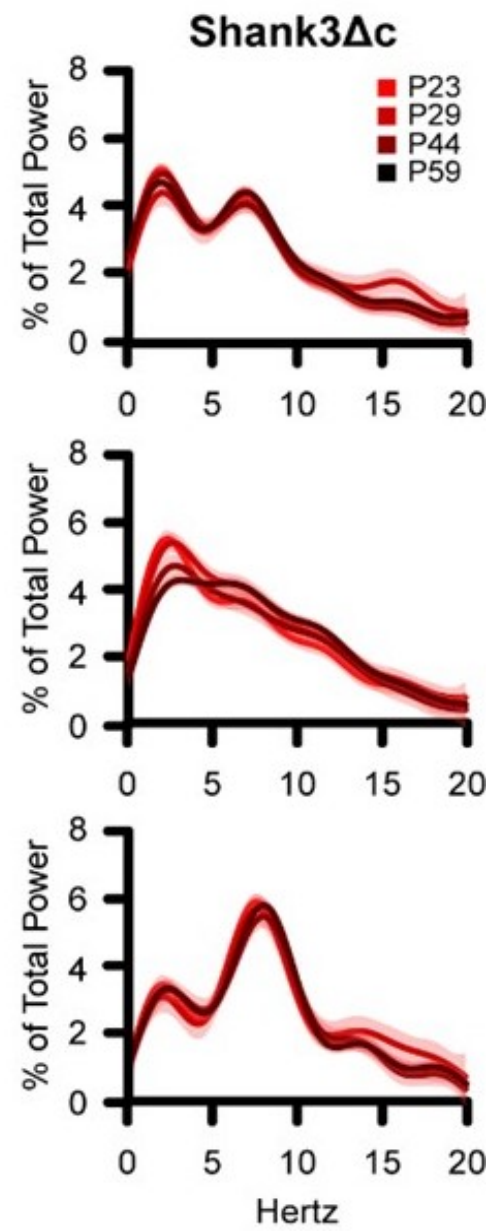

Figure 3: Spectral power changes across development in WT and Shank3 ${ }^{\Delta C}$ mice. Fourier transformed (FFT) EEG spectral power during the 12 hours of the light period (dark period yielded the same results). The rows represent wakefulness (A), NREM sleep (B), and REM sleep (C). EEG spectral power in the light period was normalized as a percentage of total statespecific EEG power in wild-type (left) or Shank $3^{\Delta C}$ mice at $P 23$ $\left(n=6\right.$ WT, 8 Shank $\left.3^{\Delta C}\right)$, P29 ( $n=7$ WT, 7 Shank3 $\left.{ }^{\Delta C}\right), P 44(n=7$ WT, 6 Shank $\left.^{\Delta C}\right)$, P59 $(n=7$ WT, 8 Shank $3^{\Delta C}$ ). Spectra are graphed as smooth lines, and significance is indicated by $95 \%$ confidence intervals.

Reduced latency to sleep following sleep loss is a developmentally acquired response that is absent in Shank3 ${ }^{\Delta \mathrm{C}}$ mice.

We previously reported that adult Shank $3^{\Delta C}$ mice show an increase latency to fall asleep sleep deprivation (SD). To better understand how this may develop, we characterized the homeostatic response to SD at P24, P30, P45 and P60 (3 hours, starting at lights on) and recorded changes in sleep/wake architecture and EEGs during the remaining 21 hours. Homeostatic increases in sleepiness due to SD in mammals is most accurately measured by changes in NREM EEG delta power $(0.5-4 \mathrm{~Hz})$; a process detectable by the third postnatal week in rodents $8,13,14$. Following 3 hours of SD, we found that WT mice at P24 and at P30 accumulate and discharge of NREM delta power in response to SD in the same way as adults (Figure 4A). However, the latency to fall asleep is significantly longer at P24 than P30 ( $p=0.02$, Figure 4B; Figure 4-Figure supplement 2). This data suggests that some components of the normal response to sleep loss continue to mature after P24, although EEG measures of sleep pressure are fully developed by P24.

At P24 and P30, Shank3 ${ }^{\Delta C}$ mice show no difference in discharge of NREM delta activity following SD relative to their WT littermates, similar to adult animals at P90 (Figure 4A). At P24 Shank3 ${ }^{\Delta C}$ mice also show no difference in latency to fall asleep following SD. However, at P30, Shank3 ${ }^{\Delta C}$ mice display an increased latency to NREM sleep in Shank3 ${ }^{\Delta C}$ relative to WT mice $(p=0.04$ (Figure 4B). Latency to the first bout of REM sleep is not affected (Figure 4-Figure supplement 1). Therefore, the net result is a reduction of NREM sleep. At later time points (P45 and P60), we don't detect differences in latency to sleep across genotypes, despite the fact that Shank3 ${ }^{\Delta C}$ mice display the same latency to sleep regardless of age (Figure 4-Figure supplement 1;

Figure 4-Figure supplement 2). The lack of differences is driven by an increase in latency to fall asleep in the WT and could be explained by either a better ability to stay awake following 3-hours of SD as animals get older (SD experiments in adults are traditionally 5-6 hours), differences in the response to SD during adolescence or long-terms effects of being isolated after electrode placement. To test the effect of SD length on latency to fall asleep in adult animals, we compared adult P90 animals after 3-hours or 5-hours of SD sleep deprivation (Figure 4-Figure supplement 1). We show that 3-hours of SD are sufficient to observe the decrease in 

available under aCC-BY 4.0 International license.

latency to NREM sleep in adult WT. Interestingly, the difference in latency to fall asleep between mutants and WT is larger following 3 hours of SD than 5 hours of SD, further supporting that this feature is independent from homeostatic EEG delta power accumulation (sleepiness). Given that Shank $3^{\Delta C}$ mice display the same response to SD at all ages, the absence of differences in sleep latency between at P45 and P60 are driven either by a normal increase in latency to fall asleep during adolescence or due to heightened stress after a month of social isolation in the WT. Regardless of the cause, Shank ${ }^{\Delta C}$ mice seem unable to adjust how long it takes to fall asleep in response to SD as they develop.

Figure 4: Shank3 ${ }^{\Delta C}$ mice fail to decrease sleep latency in response to sleep need at P30. A. Normalized NREM delta (05-4 Hz) power during recovery sleep after 3 hours of sleep deprivation during the light period (LP) relative to delta power at baseline. B. Latency to the first bout of NREM or REM sleep following 3 hours of sleep deprivation. P24 ( $n=7$ WT, 8 Shank3 $\left.{ }^{\Delta C}\right)$, P30 ( $=7$ WT, 8 Shank3 ${ }^{\Delta C}$ ) mice. * t-test p-value $<0.05$
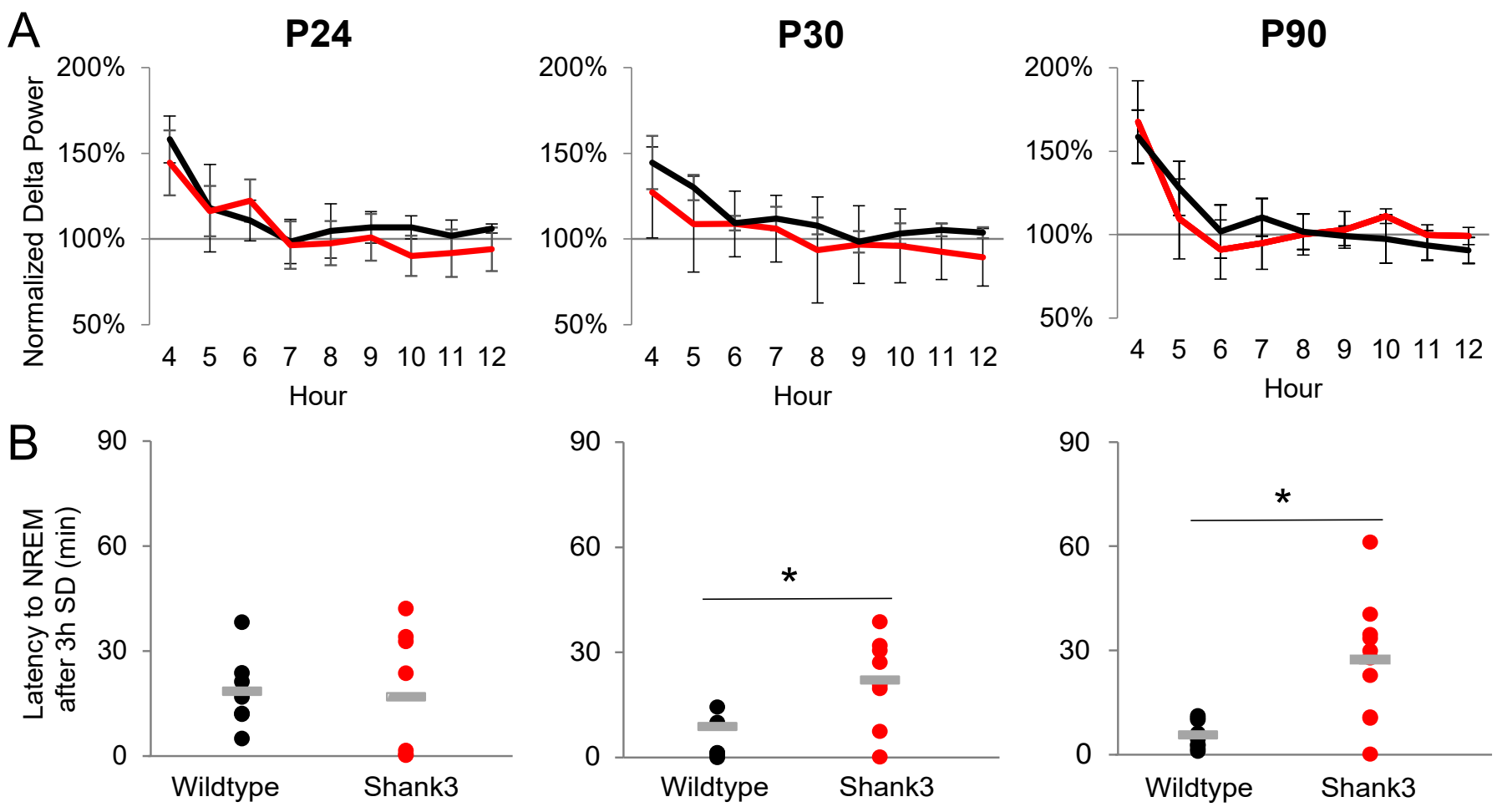

\section{DISCUSSION}

Sleep patterns in children with autism spectrum disorder (ASD) diverge from typical development early in life, but little is known about the underlying causes. To begin to address this question, we here present the first longitudinal trajectory study of postnatal sleep development in the Shank ${ }^{\Delta C}$ ASD mouse model. Our studies highlight that several features of normal sleep are still maturing between P23 and P30 in mice. At 23 days of life, mice have acquired $70 \%$ of their maximal brain volume and are equivalent to a 9-month-old infant based on brain size. At P30, mice have reached $80 \%$ of their maximal brain volume and are equivalent to an 18 month-old toddler ${ }^{10}$. We find that normal sleep at P23 occurs at a higher proportion in the active phase (night for mice) and is less consolidated (the bouts are shorter). The homeostatic response to sleep loss is also different from that of adulthood; at P23, mice take almost 3 times as long to fall asleep following sleep deprivation than they do at P30. Thus, less consolidated sleep and taking longer to fall asleep are normal features of sleep in early life. We find that Shank ${ }^{\Delta C}$ mice sleep less as early as P23, a phenotype they maintain throughout their lives. Despite sleeping less, Shank $3^{\Delta C}$ mice have larger amounts of REM sleep, specially at P23. In human brain development, the proportion of REM relative to NREM sleep is greater earlier in development and dramatically declines upon maturation ${ }^{15}$. Thus, larger amounts of REM suggest that the brain in Shank $3^{\Delta C}$ mice is in a more immature state relative to typically developing siblings. Consolidation of 
bioRxiv preprint doi: https://doi org/10.1101/2021.03 10.434728; this version posted March 10, 2021. The copyright holder for this preprint (which was not certified by peer review) is the author/funder, who has granted bioRxiv a license to display the preprint in perpetuity. It is made available under aCC-BY 4.0 International license.

NREM sleep into longer bouts is also normal feature of brain development. As expected from a more immature brain state, Shank $3^{\Delta C}$ mice show shorter NREM bouts. The increased REM activity in mutants may arises from an inability to sustain NREM sleep for longer periods of time, i.e., a failure to consolidate sleep. This in turn could underlie another common feature of the ASD sleep phenotype: sleep fragmentation.

EEG spectral analyses show that WT animals overall display more dynamic changes than mutants over time, especially during wake and REM sleep. A characteristic feature of the wake and REM sleep WT EEG spectra at P23 is the presence of a more prominent theta peak. Theta rhythms are mostly hippocampal, and therefore, its disappearance at P30 could be explained by an increase in cortical thickness as the animals age. The fact that Shank ${ }^{\Delta C}$ do not show a change in theta during wake or REM sleep over time may therefore indicate cortical overgrowth at P23, consistent with the early timing of brain overgrowth observed in infants and toddlers that go on the be diagnosed with $\mathrm{ASD}^{16}$. More studies will be needed to verify this hypothesis. Nonetheless our data suggest that a reduction of theta power in wake in infancy could serve as an early biomarker of ASD. Interestingly, Shank ${ }^{\Delta C}$ mice also show a reduction in power on the delta frequency range in NREM sleep as they age, suggesting a progressive deterioration in long-range connectivity of the network that underlies slowwave oscillations in NREM sleep which can explain why sleep problems are reported to worsen over time in ASD.

Taking longer to fall asleep, one of the more salient aspects of the Shank $3^{\Delta \mathrm{C}}$ adult phenotype, is also a defining characteristic of the clinical ASD sleep phenotype. Latency to sleep onset can only be reliably measured following sleep deprivation, to make sure that all animals are under comparable sleep pressure. Our study shows that this delay in sleep onset in Shank $3^{\Delta C}$ arises from a failure to develop a proper response to sleep between 24 and 30 days of age. This deficit arises in the absence of problems accumulating sleep pressure (sleepiness), at least as measured by increase in delta power in response to sleep deprivation. In other words, it is a failure of being able to fall asleep quickly despite being sleepy, or reminiscent of being 'over-tired'. Our studies indicate that this response is normal at young ages. At P23, WT animals take a longer time to fall asleep following sleep loss, but gradually develop the ability to respond to sleep loss by falling asleep faster. In contrast, Shank $3^{\Delta \mathrm{C}}$ mice display the same latency to fall asleep at all ages. In other words, they maintain an 'infant' response to SD throughout their lives. Our findings regarding the sleep homeostatic response parallel the immature features of baseline sleep we described above and indicate a mis-regulation of normal sleep development in Shank $3^{\Delta C}$ mice.

Although the Shank $3^{\Delta C}$ sleep phenotype can be considered immature, it may not necessarily arise from a delay in maturation, and may in contrast arise from certain aspects of the sleep cycle maturing too early or too fast. For example, Shank $3^{\Delta C}$ mutants showing a more mature diurnal/nocturnal distribution of sleep/wake at P23. The notion that an early maturation of sleep/wake distribution may explain the eventual failure to develop a proper response sleep loss may seem counterintuitive. However, it is well supported by our current understanding of sleep regulation. The two-process model of sleep regulation states that when and how much we sleep is determined by the interaction of two processes: the circadian clock and the sleep homeostat ${ }^{17}$. The circadian clock determines the timing of activity during the 24-hour day and is thought to promote arousal during the active phase. The sleep homeostat tracks sleep pressure in response to time spent awake and promotes sleep in response to sleepiness. Both processes mature after the second postnatal week in rodents ${ }^{18}$, although circadian regulation of sleep timing is known to emerge earlier than the ability to track sleep pressure. Based on our observations in WT animals, both regulatory mechanisms may mature independently until the $3^{\text {rd }}$ postnatal week, in which the circadian and hemostatic mechanisms may align, then misalign during adolescence, and eventually produce the typical response to sleep loss we observe in adult animals. If the response to circadian input matures too rapidly, one risks too much arousing input from the clock. Excess arousal input from the clock may in turn cause a failure of both processes to crosstalk effectively and explains several features of the Shank $3^{\Delta \mathrm{C}}$ sleep phenotype, namely: reduced total sleep time, earlier emergence of the diurnal/nocturnal distribution of sleep/wake, shorter NREM sleep bouts, reduced delta power in NREM sleep and delay in sleep onset following sleep deprivation. A more careful examination of the development of the mechanisms and brain regions that regulate arousal and how they interact with those that promote sleep during the third and fourth postnatal week is warranted to support this hypothesis. 
Overall, our results support a reduction of sleep as a core aspect of ASD, while highlighting a period during early life in which the abnormal sleep phenotype fully emerges and may be amenable to intervention. Our results support an excessive arousing input from the circadian clock emerging too early in development as a plausible mechanistic explanation for the observed Shank $3^{\Delta C}$ sleep phenotype. Further replication of our findings in other mouse models of ASD, as well as objective longitudinal studies of sleep in clinical cohorts will be necessary to determine which of the features we defined applies to the general ASD population, and which may be specific to the mutation we are modeling.

\section{MATERIALS AND METHODS}

Animals: Mice were bred as previously described, and housed at $24 \pm 1^{\circ} \mathrm{C}$ on a $12: 12 \mathrm{hr}$ light: dark cycle (unless otherwise specified) with food and water ad libitum. All experimental procedures were approved by the Institutional Care and Use Committee of Washington State University and conducted in accordance with National Research Council guidelines and regulations for experiments in live animals.

Surgical procedures: At 18 days old, male mice $(n=64)$ were placed under isoflurane anesthesia and stereotaxically implanted with four electroencephalographic (EEG) and two electromyographic (EMG) electrodes, according to previously published methods17. Briefly, four stainless steel wire loop electrodes were placed contralaterally over frontal (2) and parietal (2) cortices, and EMG electrodes were inserted bilaterally into the nuchal muscles. Adult mice $(n=10$, approximately 80 days old) were also implanted with four stainless steel screw electrodes (BC-002MP188, Bellcan International Corp, Hialeah, FL) as described above. To prevent damage to implants, instrumented mice were housed individually from surgery to the completion of final recording. Mice were allowed a minimum of 3 days of recovery from surgery before habituation to the recording environment.

Sleep phenotyping: Sleep recordings were conducted in 23-60-day-old male mice using a longitudinal design. Three days after surgery, mice were connected to a lightweight, flexible tether and allowed two days to habituate to the recording environment. At 23 days old, mice underwent $24 \mathrm{hr}$ undisturbed baseline EEG and EMG recording beginning at light onset (hour 1). The following day (P24), mice were sleep deprived for $3 \mathrm{hr}$ (hours 1-3) via gentle handling starting at light onset according to previously published methods7. Mice were allowed $21 \mathrm{hr}$ of recovery sleep (hours 4-12 of the light period and hours 13-24 of the dark period). A total of four $48 \mathrm{hr}$ recordings were repeated when mice were 29-30 days old, 44-45 days old, and 59-60 days old, respectively. Two $48 \mathrm{~h}$ sleep recordings in a separate cohort of adult (approximately 90 day old) mice were conducted with each animal receiving a single $3 \mathrm{~h}$ and a single $5 \mathrm{~h}$ sleep deprivation session, spaced 5 days apart.

EEG/EMG data acquisition and analysis: EEG and EMG data in animals 23-60 days old were collected with Grass 7 polygraph hardware (Natus Medical Incorporated, Pleasanton, CA) via a light-weight, counterbalanced cable, amplified, and digitized at $256 \mathrm{~Hz}$ using VitalRecorder acquisition software (SleepSign for Animal, Kissei Comtec Co., LTD, Nagano, Japan). EEG and EMG data in animals 90 days old were collected with Intan RHD2000 Interface using INTAN recording hardware (16-channel RHD USB Recording System, Intan Technologies, Los Angeles CA. EEG and EMG data were recorded at $1 \mathrm{kS} / \mathrm{s}$ with hardware amplification cutoff at $0.01 \mathrm{~Hz}$, lower and upper bandwidths at $0.10 \mathrm{~Hz}$ and $0.2 \mathrm{kHz}$, and notch filtering at $60 \mathrm{~Hz}$. Recordings were downsampled to $250 \mathrm{~Hz}$ using a custom Matlab script, and exported for manual scoring via SleepSign for Animal as previously described. Sleep states and wakefulness were determined by visual inspection of the EEG waveform and EMG activity, and vigilance states were assigned in $4 \mathrm{~s}$ increments (epochs). Latency to NREM or REM sleep after sleep deprivation was calculated as previously described. The EEG was subjected to fast Fourier transform (FFT) resulting in a power spectrum from 0-20 Hz (P23-P60) or 0-50 Hz (P90) with 0.5 $\mathrm{Hz}$ bins. Twelve-hour light period spectra were generated as previously described (Ingiosi et al 2019 ), from 0.5 $\mathrm{Hz}$ spectral bins expressed as a percentage of the sum of total state-specific EEG power $(0-20 \mathrm{~Hz}$ or $0-50 \mathrm{~Hz}$ respectively). Spectra are displayed as smooth curves with $95 \%$ confidence intervals, as generated using Generalized Additive Models using the R package mgcv (v.1.8-31). Hourly NREM delta power analysis during the 9 hours following SD was calculated from total power in delta range normalized to the average NREM power delta band from the last $4 \mathrm{~h}$ of baseline (hours 9-12). EEG epochs containing artifacts, and recordings with excessive EEG artifacts were excluded from spectral analysis. Statistical analyses were conducted as 
previously described using SPSS for Windows (IBM Corporation Armonk, NY), RStudio (v. 1.3.1056, RStudio, Boston, MA), and MS Excel 365 (2011, build 13426.20308, Microsoft, Redmond, WA). Non-continuous time-instate, bout, and latency data are plotted as individual points with a gray bar indicating the group mean.

Genotype and age-wise comparisons for dot plots were made using unpaired two-tailed t-tests with significance threshold set at $p \leq 0.05$. Hourly time-in state data are presented as means \pm standard error of the mean (SEM). Recordings in which vigilance state was obscured by extensive periods of artifact or signal loss were excluded from analysis. Outliers that fell outside \pm two standard deviations from the group mean were excluded from analysis.

Addressing Sex as a Biological Variable: This study expands on previous findings in male animals, for this reason all animals in this study are male.

\section{Funding \\ K01NS104172 NIH/NINDS to Peixoto L.}

\section{ACKNOWLEDGEMENTS}

We thank Dr. Marcos Frank for valuable discussions. We thank Dr. Paul Worley for providing the Shank3 mutant mouse line.

\section{Ethics}

Animal experimentation: This study was performed in strict accordance with the recommendations of the Guide for the Care and Use of Laboratory Animals of the Nation Institutes of Health. All of the animals were handled according to protocols approved by the Washington State University Institutional Animal Care and Use Committee (\#04704 and \#04705).

\section{COMPETING INTERESTS}

The authors have no competing interests to declare. 


\section{Supplementary materials}

Figure 2 - figure supplement 1. Increased REM sleep in young Shank $3^{\Delta C}$ mice is distributed across the light phase under baseline conditions. The rows represent vigilance states of wakefulness (top), NREM sleep (middle), and REM sleep (bottom). Time in wakefulness (A), NREM sleep (B), and REM sleep (C) during baseline 24-hour recordings is shown as a percentage of recording time per hour. Sleep was recorded at P23 $\left(n=7\right.$ WT, 8 Shank3 $\left.{ }^{\Delta C}\right)$, P29 $\left(n=7\right.$ WT, 8 Shank3 $\left.{ }^{\Delta C}\right)$, P44 $\left(n=8\right.$ WT, 10 Shank3 $\left.{ }^{\Delta C}\right)$, P59 $\left(n=8\right.$ WT, 8 Shank3 $\left.{ }^{\Delta C}\right)$ mice. Repeated measures-ANOVA, main effect of genotype * $p<0.05$. WT in black, Mutants in red.
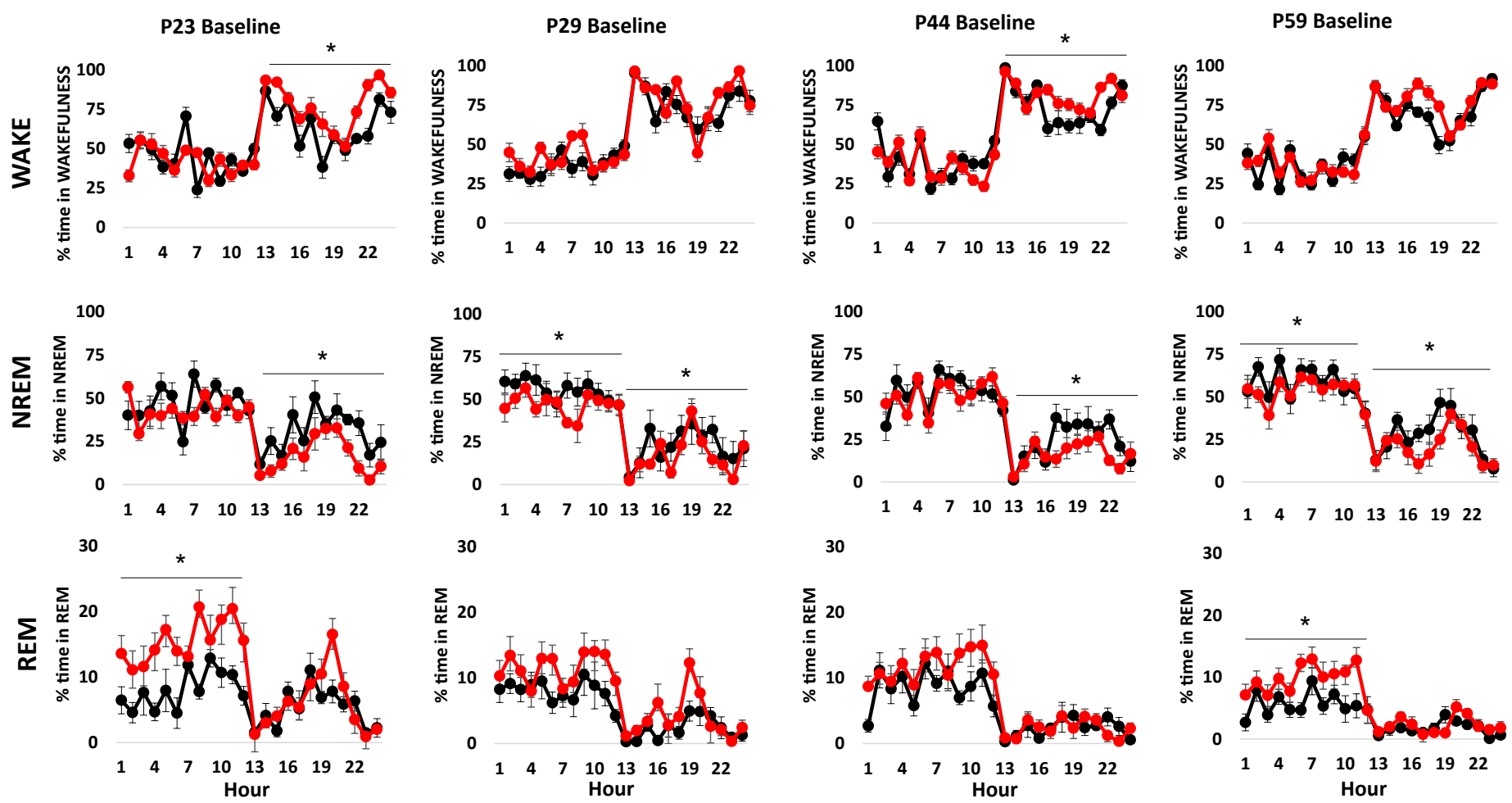
Figure 2- figure supplement 2. Age-dependent loss of baseline sleep time in Shank ${ }^{\Delta C}$ mice is driven by reduced duration of NREM bouts. The rows represent average number (A-C) and duration (D-F) of bouts of wakefulness $(A, D)$, NREM sleep $(B, E)$, and REM sleep $(C, F)$ during baseline $12 \mathrm{~h}$ light (white) and dark (gray) periods. Sleep was recorded at P23 ( $n=7$ WT, 8 Shank3 $\left.{ }^{\Delta C}\right), P 29\left(n=7\right.$ WT, 8 Shank3 $\left.{ }^{\Delta C}\right), P 44(n=8$ WT, 10 Shank3 $\left.{ }^{\Delta C}\right)$, P59 $\left(n=8\right.$ WT, 8 Shank3 $\left.{ }^{\Delta C}\right)$ mice. * $p$-value $<0.05$, repeated measures ANOVA. WT in black, Mutants in red.

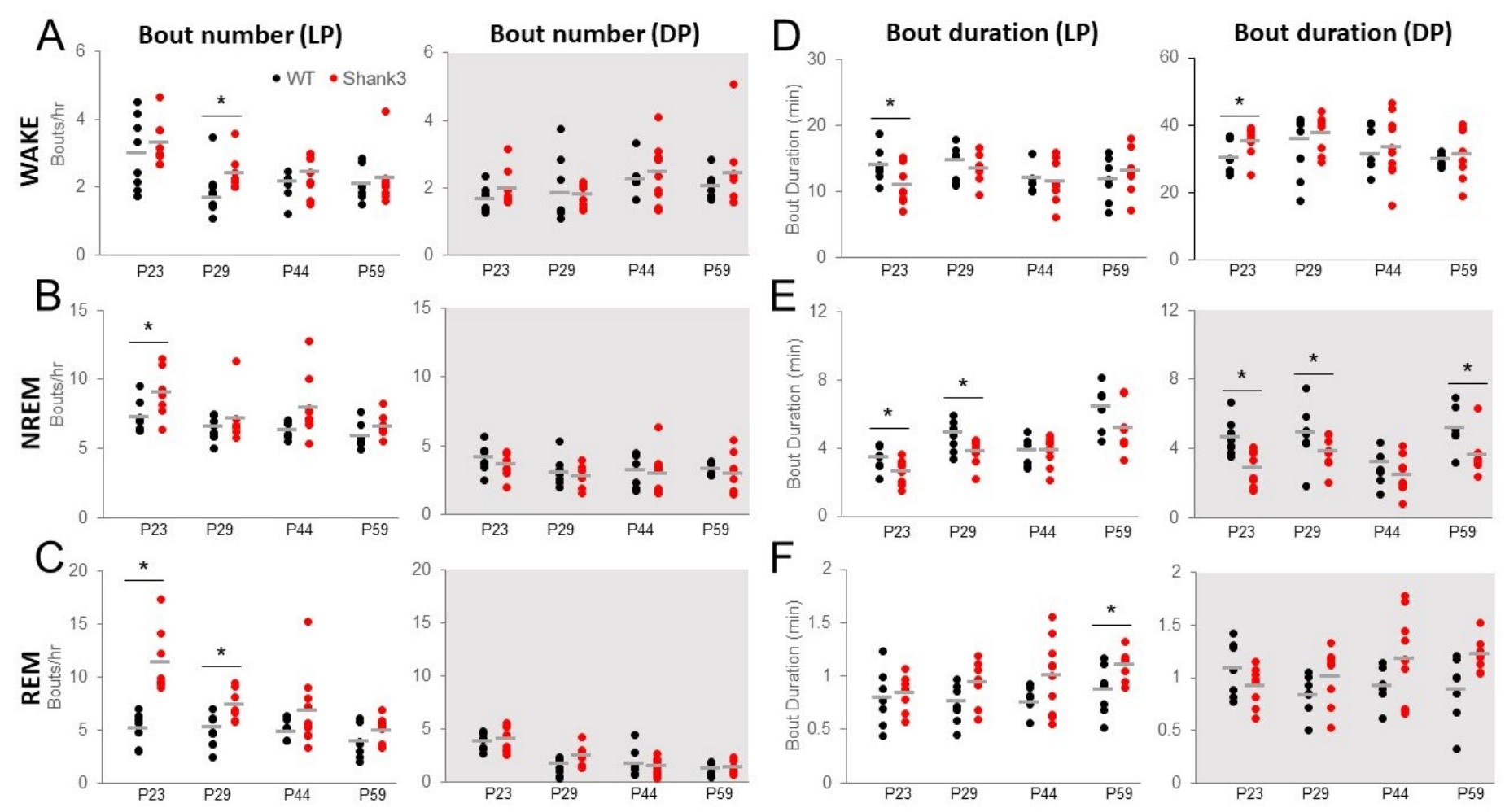


Figure 3- figure supplement 1: Progressive changes in NREM delta activity and peak REM theta activity in Shank3 ${ }^{\Delta C}$ mice. The rows represent vigilance states of wakefulness (top), NREM sleep (middle), and REM sleep (bottom). EEG spectral power in the light period normalized as a percentage of total state-specific EEG power in wild-type (left) or Shank3 ${ }^{\Delta C}$ mice at P23 ( $n=6$ WT, 8 Shank3 $\left.{ }^{\Delta C}\right), P 29\left(n=7\right.$ WT, 7 Shank3 $\left.{ }^{\Delta C}\right), P 44(n=7$ WT, 6 Shank3 $\left.{ }^{\Delta C}\right)$, P59 ( $n=7$ WT, 8 Shank3 $\left.{ }^{\Delta C}\right)$. Spectra are graphed as smooth lines, and significance is indicated by non-overlap of 95\% confidence intervals. WT in black, Mutants in red.
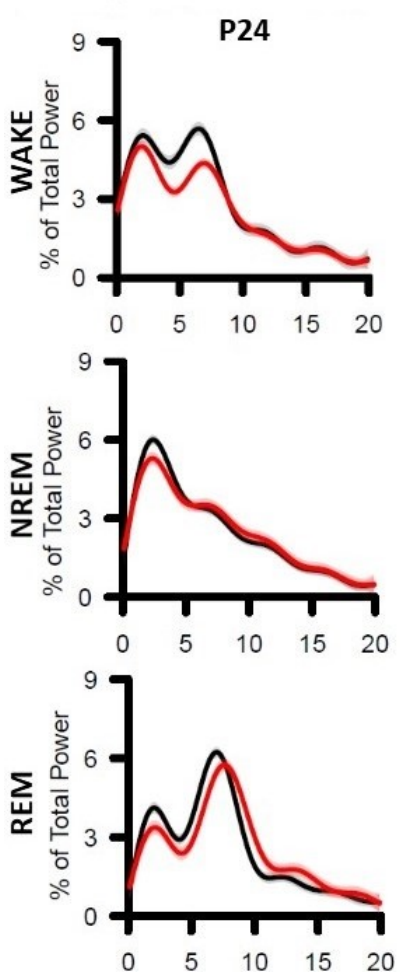
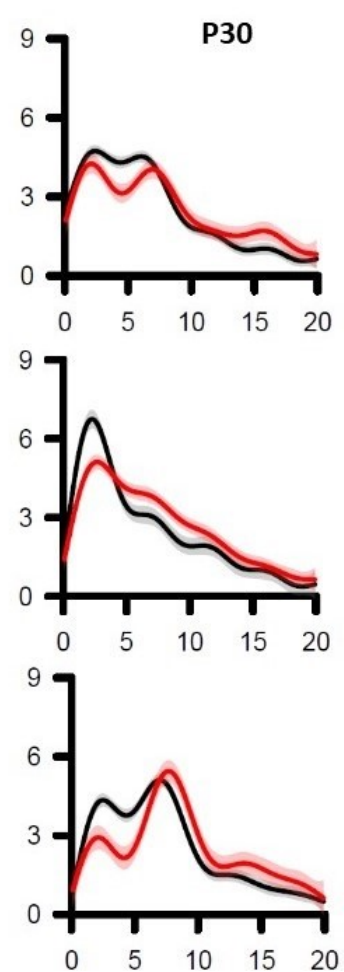
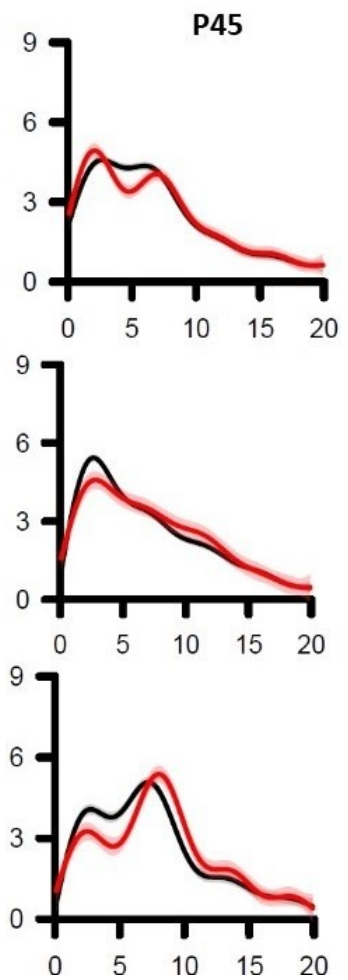

P60
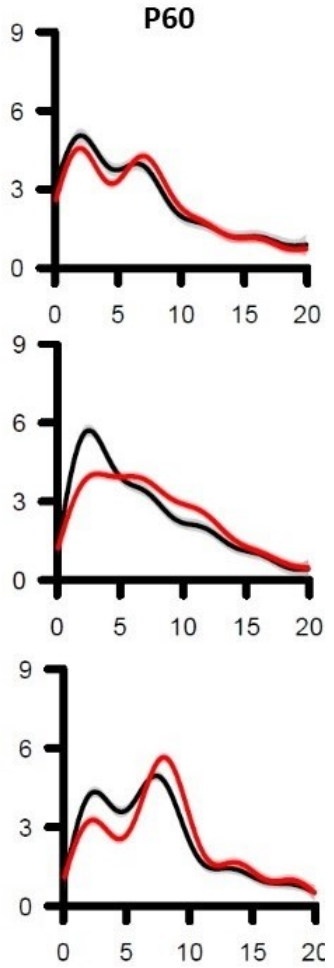
Figure 4- figure supplement 1 . Sleep latency is increased at $P 30$ and P90 in Shank3 ${ }^{\Delta C}$ mice. Latency to the first bout of NREM sleep following $3 \mathrm{~h}$ of sleep deprivation at P24 $\left(n=7\right.$ WT, 8 Shank3 $\left.{ }^{\Delta C}\right), P 30(n=7$ WT, 8 Shank3 $\left.{ }^{\Delta C}\right), P 45\left(n=8\right.$ WT, 9 Shank $\left.3^{\Delta C}\right), P 60\left(n=7\right.$ WT, 7 Shank3 $\left.{ }^{\Delta C}\right)$, and P90 $\left(n=5\right.$ WT, 5 Shank $\left.3^{\Delta C}\right)$ mice. ${ }^{*} p-$ value $<0.05$, two-tailed unpaired t-tests. WT in black, Mutants in red.

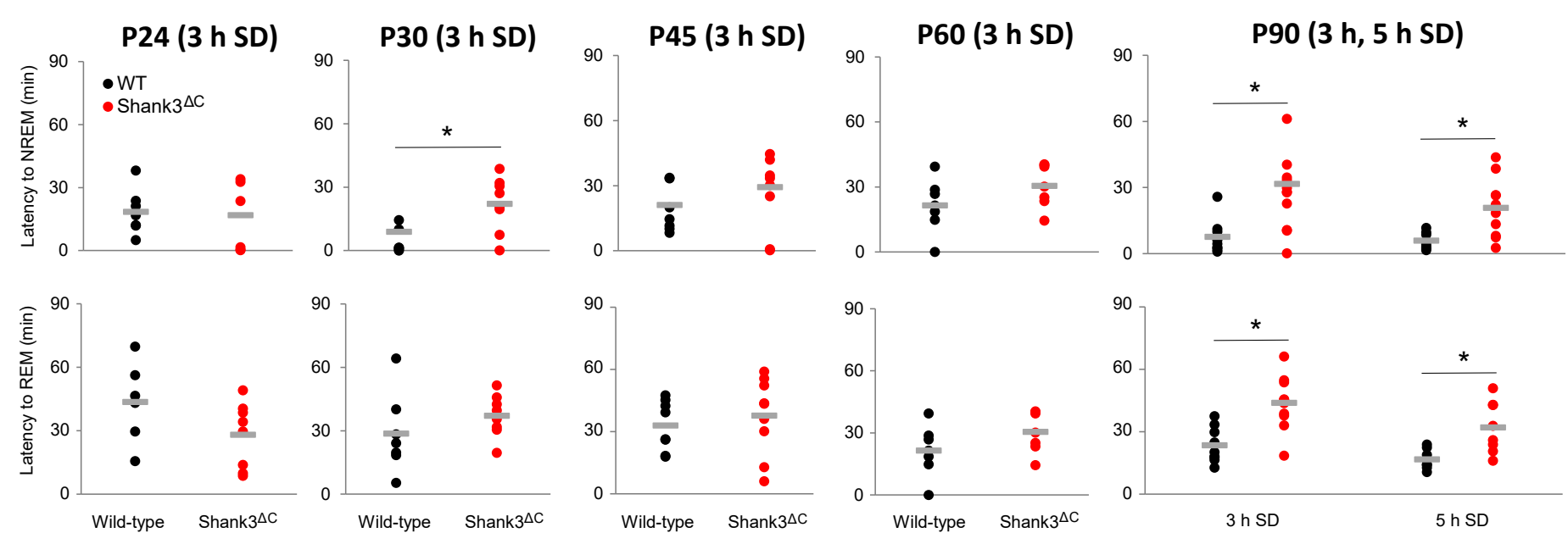

Figure 4- figure supplement 2. Latency to fall asleep changes over time in WT but not in Shank3 ${ }^{\Delta C}$ mice. Difference in latency to the first bout of NREM sleep following $3 \mathrm{~h}$ of sleep deprivation in minutes across timepoints. P24 ( $n=7$ WT, 8 Shank $\left.{ }^{\Delta C}\right), P 30\left(n=7\right.$ WT, 8 Shank3 $\left.{ }^{\Delta C}\right), P 45\left(n=8\right.$ WT, 9 Shank3 $\left.{ }^{\Delta C}\right), P 60(n=7$ WT, 7 Shank $\left.3^{\Delta C}\right)$, and $P 90\left(n=5\right.$ WT, 5 Shank $\left.3^{\Delta C}\right)$ mice. * $p$-value $<0.05$, two-tailed unpaired t-tests.

\begin{tabular}{|c|c|c|c|c|}
\hline \multicolumn{5}{|c|}{ Differences in latency to fall asleep after 3 hours of SD across time-points } \\
\hline \multicolumn{5}{|c|}{ Within-genotype comparisons } \\
\hline \multicolumn{5}{|c|}{ Wild-type } \\
\hline & Difference in latency to & Fold Change & $\mathrm{p}$-value (t-test) & number of replicates \\
\hline P24 v. P30 & -14 & 0.239 & 0.02 & $\mathrm{n}=7$ vs $\mathrm{n}=6$ \\
\hline P30 v. P45 & 16 & 4.517 & 0.01 & $\mathrm{n}=6$ vs $\mathrm{n}=8$ \\
\hline P45 v. P60 & 0 & 1.018 & 0.95 & $\mathrm{n}=8$ vs $\mathrm{n}=7$ \\
\hline P60 v. P90 & -14 & 0.357 & 0.01 & $n=7$ vs $n=10$ \\
\hline \multicolumn{5}{|c|}{ Shank $3^{\Delta C}$} \\
\hline & Difference in latency to & Fold Change & $\mathrm{p}$-value (t-test) & number of replicates \\
\hline P24 v. P30 & 5 & 1.31 & 0.52 & $n=8$ vs $n=8$ \\
\hline P30 v. P45 & 7 & 1.323 & 0.37 & $n=8$ vs $n=9$ \\
\hline P45 v. P60 & 1 & 1.044 & 0.87 & $n=9$ vs $n=7$ \\
\hline P60 v. P90 & -3 & 0.894 & 0.65 & $n=7$ vs $n=10$ \\
\hline
\end{tabular}




\section{REFERENCES}

1. Maxwell-Horn, A. \& Malow, B. A. Sleep in Autism. Semin Neurol 37, 413-418 (2017).

2. Souders, M. C. et al. Sleep in Children with Autism Spectrum Disorder. Curr Psychiatry Rep 19, 34 (2017).

3. Hodge, D., Carollo, T. M., Lewin, M., Hoffman, C. D. \& Sweeney, D. P. Sleep patterns in children with and without autism spectrum disorders: developmental comparisons. Res Dev Disabil 35, 1631-1638 (2014).

4. MacDuffie, K. E. et al. Sleep Problems and Trajectories of Restricted and Repetitive Behaviors in Children with Neurodevelopmental Disabilities. J Autism Dev Disord (2020) doi:10.1007/s10803-020-04438-y.

5. MacDuffie, K. E. et al. Sleep Onset Problems and Subcortical Development in Infants Later Diagnosed With Autism Spectrum Disorder. Am J Psychiatry 177, 518-525 (2020).

6. Rylaarsdam, L. \& Guemez-Gamboa, A. Genetic Causes and Modifiers of Autism Spectrum Disorder. Front Cell Neurosci 13, 385 (2019).

7. Ingiosi, A. M. et al. Shank3 modulates sleep and expression of circadian transcription factors. Elife 8, (2019).

8. Nelson, A. B., Faraguna, U., Zoltan, J. T., Tononi, G. \& Cirelli, C. Sleep Patterns and Homeostatic Mechanisms in Adolescent Mice. Brain Sci 3, 318-343 (2013).

9. Rensing, N., Moy, B., Friedman, J. L., Galindo, R. \& Wong, M. Longitudinal analysis of developmental changes in electroencephalography patterns and sleep-wake states of the neonatal mouse. PLoS ONE 13, e0207031 (2018).

10. Workman, A. D., Charvet, C. J., Clancy, B., Darlington, R. B. \& Finlay, B. L. Modeling Transformations of Neurodevelopmental Sequences across Mammalian Species. J. Neurosci. 33, 7368-7383 (2013).

11. Zwaigenbaum, L. et al. Behavioral manifestations of autism in the first year of life. Int J Dev Neurosci 23, 143-152 (2005).

12. Frank, M. G. \& Heller, H. C. Development of REM and slow wave sleep in the rat. Am. J. Physiol. 272, R1792-1799 (1997).

13. Franken, P., Chollet, D. \& Tafti, M. The homeostatic regulation of sleep need is under genetic control. $J$. Neurosci. 21, 2610-2621 (2001).

14. Frank, M. G., Morrissette, R. \& Heller, H. C. Effects of sleep deprivation in neonatal rats. Am. J. Physiol. 275, R148-157 (1998).

15. Roffwarg, H. P., Muzio, J. N. \& Dement, W. C. Ontogenetic development of the human sleep-dream cycle. Science 152, 604-619 (1966).

16. Hazlett, H. C. et al. Early Brain Overgrowth in Autism Associated with an Increase in Cortical Surface Area Before Age 2. Arch Gen Psychiatry 68, 467-476 (2011).

17. Borbély, A. A. A two process model of sleep regulation. Hum Neurobiol 1, 195-204 (1982).

18. Frank, M. G., Ruby, N. F., Heller, H. C. \& Franken, P. Development of Circadian Sleep Regulation in the Rat: A Longitudinal Study Under Constant Conditions. Sleep 40, (2016). 